\title{
Bloqueo simpático lumbar
}

\author{
J.M. González Mesa, E. Otero Granados, M. del Valle Hoyos y M. Rivera \\ Unidad Atención Integral al Dolor. Hospital Universitario Virgen de La Victoria. Málaga
}

González Mesa JM, Otero Granados E, del Valle Hoyos M, Rivera M. Bloqueo simpático lumbar. Rev Soc Esp Dolor 2013; 20(6): 324-331.

\section{INTRODUCCIÓN}

En 1924 Brunn y Mandl describieron por primera vez el bloqueo percutáneo de la cadena y simpática lumbar, inspirados en el abordaje del plexo celiaco descrito por Kappis. Con esta técnica se pretendía obtener un bloqueo simpático efectivo pronóstico o terapéutico menos agresivo que la simpatectomía estándar desarrollada por Adson y Díez a principios del siglo XX (1). Pronto variantes como las descritas por Bryce Smith (1951) y Wallace (1957) hacían el procedimiento más tolerable incorporando la utilización de anestésicos locales y evitando el contacto con las apófisis transversas. En 1970 Reid describe el abordaje lateral considerado actualmente como técnica estándar.

Desde la segunda mitad del siglo pasado el bloqueo simpático lumbar fue implementado fundamentalmente por anestesiólogos en las unidades de dolor. En 1950 Bonica, Moore y Arnulf describieron la importancia del bloqueo simpático lumbar en el tratamiento de la causalgia y distrofia simpática refleja postraumática en excombatientes de la Segunda Guerra Mundial.

Con el desarrollo tecnológico se han incorporado técnicas de imagen que aportan eficacia y seguridad a la técnica original. En 1947 Duncan Alexander inyectó contraste iodado al realizar una simpatectomía química lumbar para

Recibido: 09-08-13.

Aceptado: 10-09-13. evidenciar la difusión del agente neurolítico, pero no es hasta 1976 cuando Robert Boas introduce como práctica clínica habitual la monitorización fluoroscópica continua de inyecciones de contraste iodado y alcohol en el abordaje de la cadena simpática lumbar.

En 1990, Reddy y Chitangada usaron por primera vez el TAC en el bloqueo simpático lumbar (2) logrando resultados satisfactorios $(3,4)$.

Una nueva alternativa a la neurólisis clásica aparece con la introducción de la radiofrecuencia. En 1991 Haynswoorth y Noe utilizaron por primera vez la radiofrecuencia como alternativa a la neurólisis química obteniendo un menor índice de complicaciones.

Recientemente la incorporación los bloqueos ecoguiados ha planteado un nuevo reto. En 1992 Kirvelä demuestran la posibilidad de realizar el procedimiento bajo control ecográfico aunque la dificultad técnica es notable y debe complementarse con control fluoroscópico $(5,6)$.

\section{SISTEMA NERVIOSO AUTÓNOMO SIMPÁTICO}

El sistema nervioso simpático está implicado en la génesis y perpetuación del dolor neuropático, vascular y visceral. El bloqueo de los ganglios simpáticos lumbares constituye una fuente básica de información que nos proporciona una idea certera sobre el papel del sistema autónomo en un síndrome doloroso concreto.

El sistema nervioso simpático o toracolumbar tiene su origen en la médula espinal, entre los segmentos C8 o T1. Podemos distinguir un brazo eferente que aporta flujo simpático a nervios metaméricos y esplácnicos y un brazo aferente que vehicula la sensibilidad visceral. El brazo eferente de la vía simpática se compone de dos neuronas, una preganglionar y una posganglionar (7). 
El cuerpo celular de cada neurona preganglionar se halla en el asta intermedio-lateral de la médula espinal y sus fibras atraviesan la raíz anterior de la médula hasta el correspondiente nervio raquídeo.

Estas neuronas envían sus fibras preganglionares a través del asta anterior para viajar con los nervios raquídeos. Inmediatamente después de que el nervio raquídeo abandona la columna, las fibras simpáticas preganglionares dejan el nervio somático formando el ramo comunicante blanco. Las fibras pueden sinaptar con neuronas posganglionares en el ganglio en que penetra en primer lugar, ascender o descender por la cadena ganglionar paravertebral y establecer sinapsis en otros ganglios de la misma o en un ganglio prevertebral.

La neurona posganglionar tiene su origen en uno de los ganglios de la cadena simpática paravertebral o en los plexos prevertebrales. Desde cualquiera de estos dos puntos de partida las fibras posganglionares viajan a sus destinos en los diversos órganos. Algunas vuelven a penetrar desde la cadena simpática hacia los nervios raquídeos por los ramos grises comunicantes aportando flujo simpático a los nervios metaméricos; otras son fibras viscerales (nervio esplácnico) que nacen de los ganglios laterovertebrales o de los prevertebrales y se dirigen al órgano al que están destinadas directamente o después de haber formado un plexo nervioso simpático. De estos ganglios se originan las fibras posganglionares que se dirigen a las vísceras abdominopélvicas o efectores periféricos del territorio cefálico, a través de nervios raquídeos o plexos periarteriales (Fig. 1).
El brazo aferente va a recoger sensibilidad visceral. Las fibras viscerales atraviesan la cadena simpática, mediante el ramo comunicante blanco, y llegan al nervio raquídeo. El cuerpo de la neurona está en el ganglio raquídeo, terminando en las astas posteriores.

\section{CADENA SIMPÁTICA LUMBAR. GANGLIOS SIM- PÁTICOS LUMBARES}

La porción lumbar de la cadena simpática está formada por dos cadenas ganglionares que se extienden retroperitonealmente a ambos lados de la columna vertebral desde L1 a L5. Se sitúa en el borde anterolateral de los cuerpos vertebrales lumbares por la zona interna de la inserción del músculo psoas. La cadena simpática está separada de las raíces nerviosas somáticas lumbares por cada músculo psoas y su fascia. A medida que va descendiendo la cadena se coloca delante de los cuerpos vertebrales, continuando caudalmente por detrás de los vasos iliacos primitivos con el tronco simpático pélvico.

Se describen un total de cuatro o cinco ganglios simpáticos lumbares dependiendo de la fusión de los ganglios T12 y L5. El tamaño varía entre los 3 y $5 \mathrm{~mm}$ de ancho por 10-15 mm de largo, son fusiformes. La distancia entre cada ganglio simpático lumbar y la apófisis transversa de cada vértebra es de 4 a $5 \mathrm{~cm}$.

Existen ramos comunicantes blancos para los dos primeros ganglios que se unen a los correspondientes nervios

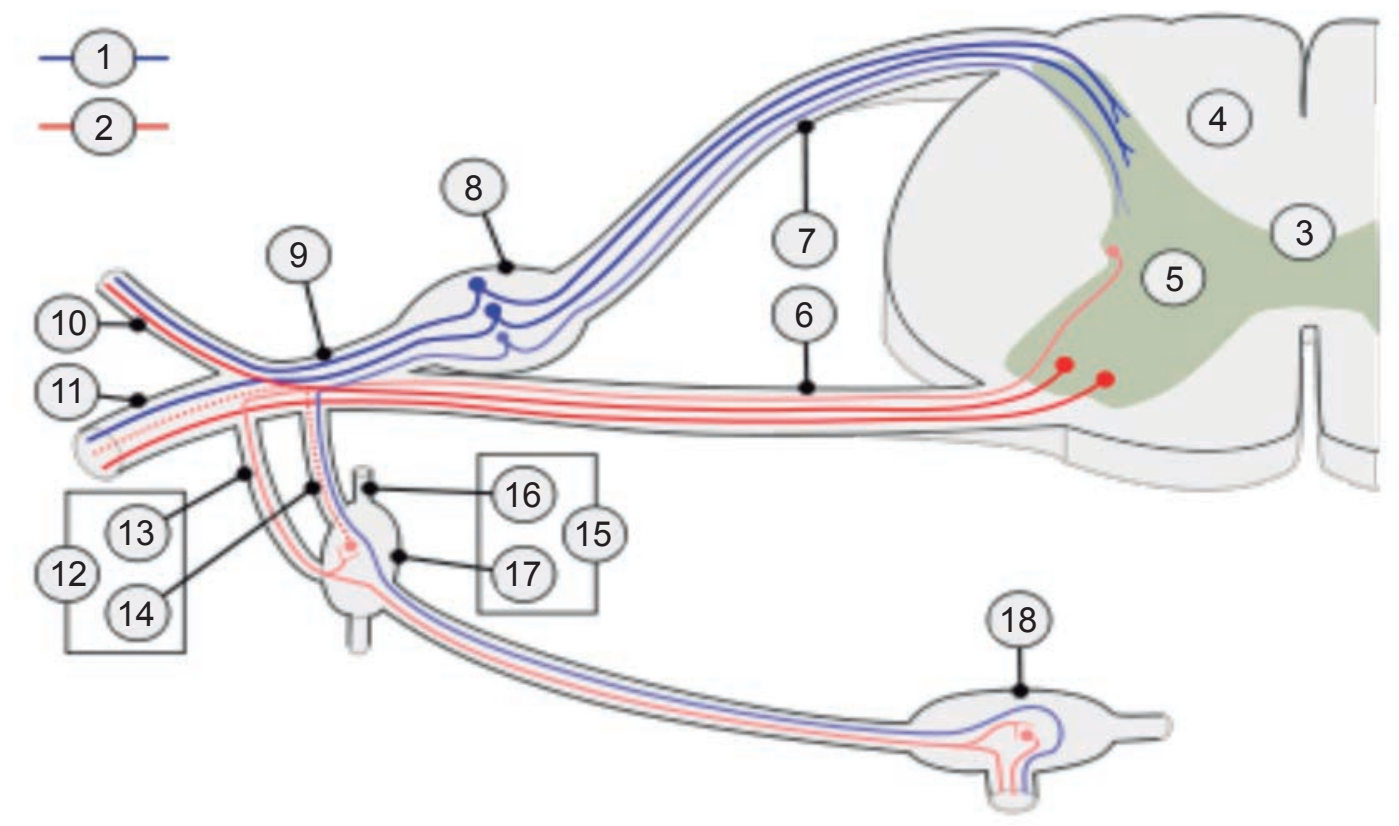

Fig. 1. Esquema de un nervio espinal y un ganglio simpático, componente del tronco simpático. Fibras: sensitivas (1) y motoras (2). Médula espinal (3): sustancia blanca (4) y sustancia gris (5). Astas de la médula: anterior (6) y posterior (7). Ganglio espinal (8). Nervio espinal (9). Ramas: dorsal (10) y ventral (11). Ramos comunicantes (12): blanco (13) y gris (14). Tronco simpático (15): ramo interganglionar (16) y ganglio paravertebral (17). Ganglio prevertebral (18). 
espinales. Son muy oblicuos y atraviesan los fascículos del músculo psoas (8). Todos los ganglios lumbares presentan ramos comunicantes grises, en disposición más o menos transversal. Los axones posganglionares salen de la cadena y forman un plexo, alrededor de las arterias iliacas, que aporta flujo simpático a los nervios femoral y obturador.

Aparte de los ramos comunicantes, los ganglios simpáticos lumbares dan lugar a cuatro nervios esplácnicos lumbares. El primero se dirige al plexo solar, el segundo al plexo intermesentérico, el tercero se sitúa delante de los vasos iliacos primitivos para llegar al plexo hipogástrico, y el cuarto nervio viene del ganglio simpático lumbar inferior situándose detrás de los vasos iliacos primitivos, para dirigirse a la pelvis, aunque da fibras a la porción inferior del plexo hipogástrico superior (9-11).

Los dos primeros nervios esplácnicos se quedan por lo tanto en el abdomen y los dos últimos se dirigen hacia la cavidad pélvica. Estos últimos se denominan nervios esplácnicos del abdomen o nervios hipogástricos de Latarjet (Fig. 2).

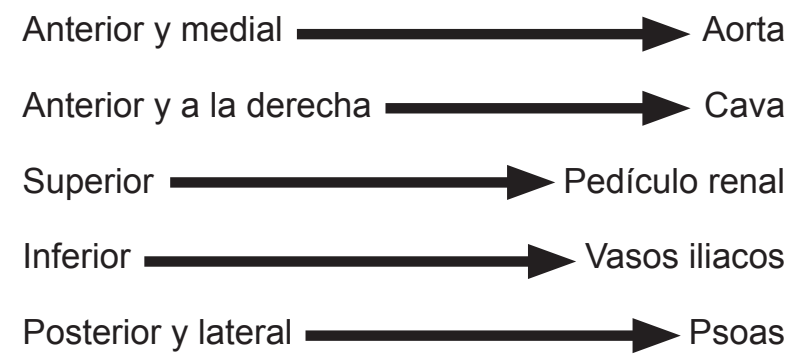

Fig. 2. Límites de cadena ganglionar lumbar.

\section{INDICACIONES (Fig. 3)}

La indicación del bloqueo simpático lumbar se basa en la observación del hecho de que en determinadas circunstancias el dolor es modulado por el sistema nervioso simpático. Sabemos que la neurona posganglionar simpática actúa no solo con función efectora sino que también recoge estímulos aferentes vehiculados a través de los nervios esplácnicos y ramos comunicantes grises.

El bloqueo simpático lumbar pretende lograr la interrupción de las eferencias simpáticas que origina la disminución de resistencia periférica con aumento de temperatura local y el stop de las aferencias viscerales de los miembros inferiores que viajan con los nervios simpáticos.

La mayor parte de la experiencia sobre el bloqueo simpático lumbar (BSL) proviene del tratamiento de vasculopatías oclusivas; también ha demostrado su utilidad en el tratamiento del dolor oncológico, el SDRC tipos I y II, síndrome poslaminectomía, miembro fantasma, herpes zóster en fase aguda y en los estadios iniciales de la neuralgia posherpética (Dobrogowski, 1995) (12-14).
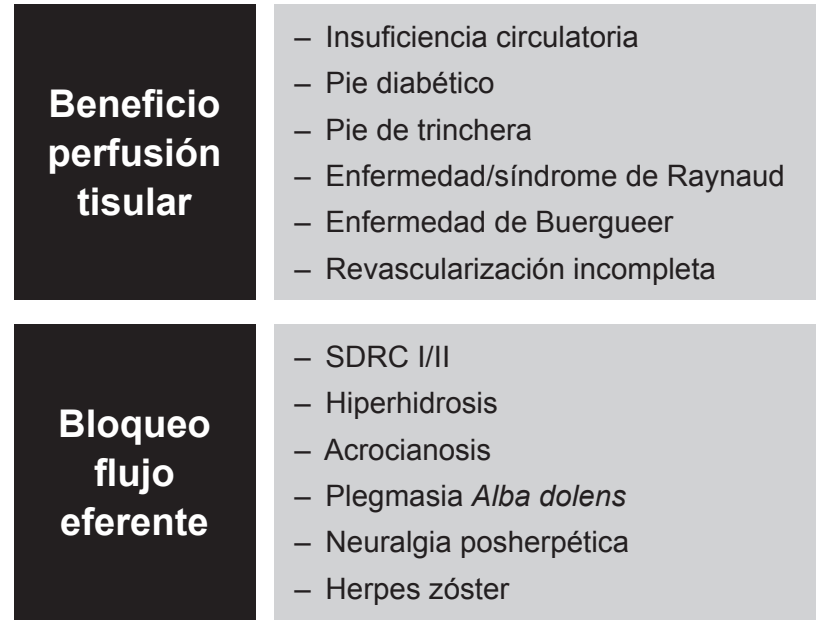

\section{Bloqueo flujo aferente}

Fig. 3. Indicaciones bloqueo simpático lumbar.

Podemos definir las indicaciones del BSL en función del objetivo primario perseguido con la simpaticolisis:

- Procesos vasculares que afectan a los miembros inferiores susceptibles de beneficiarse de la disminución de resistencias periféricas y aumento flujo sanguíneo.

- En el segundo grupo de indicaciones el objetivo es actuar sobre el flujo simpático eferente como en el caso del síndrome de dolor regional complejo (SDRC) (tipos I y II).

En todas las situaciones en las que pretendemos actuar sobre las aferencias pélvicas y de miembros inferiores cuando en el tratamiento del dolor de origen visceral o vascular (15-18).

\section{NIVELES DE RECOMENDACIÓN BASADOS EN LA EVIDENCIA. BLOQUEO SIMPÁTICO LUMBAR}

A pesar de que han sido reportados casos de simpatectomía química lumbar para un gran variedad de indicaciones, se han descrito simpatectomías químicas lumbares exitosas en el dolor agudo para el tratamiento del dolor y necrosis producido por picaduras de arañas, o el alivio de contracturas musculares secundarias a fracturas vertebrales y en casos de dolor pélvico de origen oncológico y no oncológico. Sin embargo, existen pocos estudios que puedan aportar evidencia de suficiente calidad $(19,20)$.

Este hecho debe hacernos valorar la indicación detenidamente en cada caso concreto. Para ofrecer un tratamiento intervencionista como el bloqueo simpático lumbar en una indicación no basada en la evidencia, el paciente ha debido 
seguir tratamiento conservador sin éxito y se habrá realizado un bloqueo diagnóstico. La relación riesgo-beneficio de la técnica debe ser favorable. Siempre se realizará en el medio adecuado, con los recursos suficientes y por médicos experimentados.

Existen algunos estudios prospectivos randomizados como el de Haynsworth y Noe que compara la neurólisis lumbar química frente a la térmica por radiofrecuencia, y el de Cross y Coton en dolor isquémico en reposo. Existen también series de casos de estudios retrospectivos en el tratamiento de la neuralgia posherpética.

En base a los criterios actuales podemos decir que existe recomendación favorable para el bloqueo simpático lumbar en el SDRCI y en el dolor isquémico en reposo (Tabla I).

TABLA I. GRADO DE EVIDENCIA

\begin{tabular}{lc}
\hline SDRC I & $1 \mathrm{~B}+$ \\
Dolor isquémico Raynaud & $1 \mathrm{~B} \pm$ \\
Herpes zóster y neuralgia posherpética & $1 \mathrm{C}+$ \\
\hline Grado de la recomendación bloqueo simpático lumbar: \\
- B: basada en una categoría de evidencia II. \\
Recomendación favorable \\
- C: basada en una categoría de evidencia III. \\
Recomendación favorable pero no concluyente
\end{tabular}

\section{CONTRAINDICACIONES}

Contraindicaciones absolutas serían la presencia de sangrado activo (21), diátesis hemorrágicas, infección local y sepsis.

Como contraindicaciones relativas destacarían ciertas anomalías estructurales que pueden dificultar la técnica provocando un disbalance entre riesgos y beneficios, como por ejemplo, un aneurisma de aorta abdominal.

\section{TÉCNICA}

La técnica de bloqueo simpático lumbar se puede dividir didácticamente en dos fases. En primer lugar accederemos vía percutánea al objetivo, comprobando y asegurándonos la correcta localización; posteriormente procederemos a la realización del bloqueo mediante anestésicos locales o agentes neurolíticos.

Para lograr una localización precisa y segura el bloqueo percutáneo de los ganglios y cadena simpática lumbar se ayuda de técnicas de imagen. Se han descrito bloqueos con éxito realizados con la ayuda de TAC y resonancia magnética, pero necesitan una gran infraestructura por lo que no son prácticas habituales. La visualización sonoanatómica es un reto debido a la profundidad de las estructuras a tratar y aunque también ha sido utilizada con éxito, para realizar bloqueos neurolíticos, es recomendadable acompañarla con apoyo fluoroscópico convencional (21). En la práctica clínica el acceso percutáneo es guiado mediante un arco de escopia.

La técnica de punción lateral descrita por Reid es la más frecuentemente utilizada. El abordaje clásico o paramedial, aunque habitualmente no sea el procedimiento de primera elección, es de gran utilidad a la hora de dejar catéteres para perfusión continua de anestésicos locales en la cadena simpática lumbar (22).

Es necesaria una cuidadosa preparación que comienza con la explicación al paciente de los beneficios y riesgos esperados como consecuencia del procedimiento. La técnica se realizará en quirófano o sala de $\mathrm{Rx}$ intervencionista con los medios necesarios de monitorización y de soporte vital avanzado.

En todos los casos se procederá a canalizar acceso venoso y a monitorizar al paciente según los estándares de la SEDAR. El nivel de sedación/anestesia indicado debe ser el adecuado para permitir el procedimiento, utilizando el menor número de fármacos posible, pero considerando que una sedación o analgesia inapropiada puede resultar en profundo disconfort para el paciente por falta de cooperación o respuesta excesiva al estrés.

\section{ABORDAJE PARAMEDIAL O DE MANDL}

\section{Descripción de la técnica}

Es un procedimiento doloroso y no siempre bien tolerado.

- El paciente se coloca en decúbito lateral o prono mediante fluoroscopia se marcan las apófisis espinosas de L2, L3, L4. Se traza una línea paralela a 4,5 cm y los puntos de intersección son los puntos de entrada de las agujas que se introducen con un ángulo de 70 u 80 grados hacia la línea media, hasta contactar con las apófisis transversas a 3 o $4 \mathrm{~cm}$ de la piel.

- Se retira la aguja y se dirige medial e inferiormente para que deslice por la apófisis transversa y supere el cuerpo vertebral alcanzando el retroperitoneo.

- Se repetirá el procedimiento en cada nivel. Por tanto se necesitarán tres agujas.

- Se comprobará la posición final de las agujas mediante la administración de contraste iodado control radiológico $(23,24)$.

\section{TÉCNICA LATERAL O DE REID}

Descrita en 1970 ha ido ganando popularidad hasta convertirse en la técnica de elección ya que es mejor tolerada 
y sigue una trayectoria alejada de los nervios metaméricos constituyendo una vía directa de abordaje de la cadena simpática y sus ganglios. En manos expertas en el $80 \%$ de las ocasiones es suficiente una sola aguja.

- El paciente estará colocado en decúbito prono con una almohada debajo del abdomen para rectificar la columna.

- El arco del intensificador de imagen se colocará en el lado a tratar. Con una ligera angulación cráneo-caudal podemos eliminar el doble contorno terminal del cuerpo vertebral seleccionado.

- Para la localización del punto de entrada percutáneo se oblicuará el haz de $\mathrm{Rx} 15$ o $20^{\circ}$ hacia el lado a tratar hasta que la apófisis transversa desaparezca cubierta por el cuerpo vertebral. Marcaremos como punto de entrada la intersección del borde inferior de la apófisis transversa y el cuerpo vertebral (Fig. 4). En este punto se introduce la aguja de forma perpendicular al haz de rayos o túnel visión.

- Para avanzar en profundidad el arco de escopia se colocará posición lateral. Se progresa la aguja hasta que la punta alcance el borde anterior del cuerpo vertebral.

- Mediante la técnica de pérdida de resistencia confirmaremos el paso de la fascia del músculo psoas.

- Inyectaremos medio de contraste que debe quedar en la proyección lateral como una línea en contacto con el borde anterior del cuerpo vertebral (Fig. 5). En proyección anteroposterior la punta de la aguja debe quedar un cm dentro del cuerpo vertebral y debajo del pedículo (23-25).

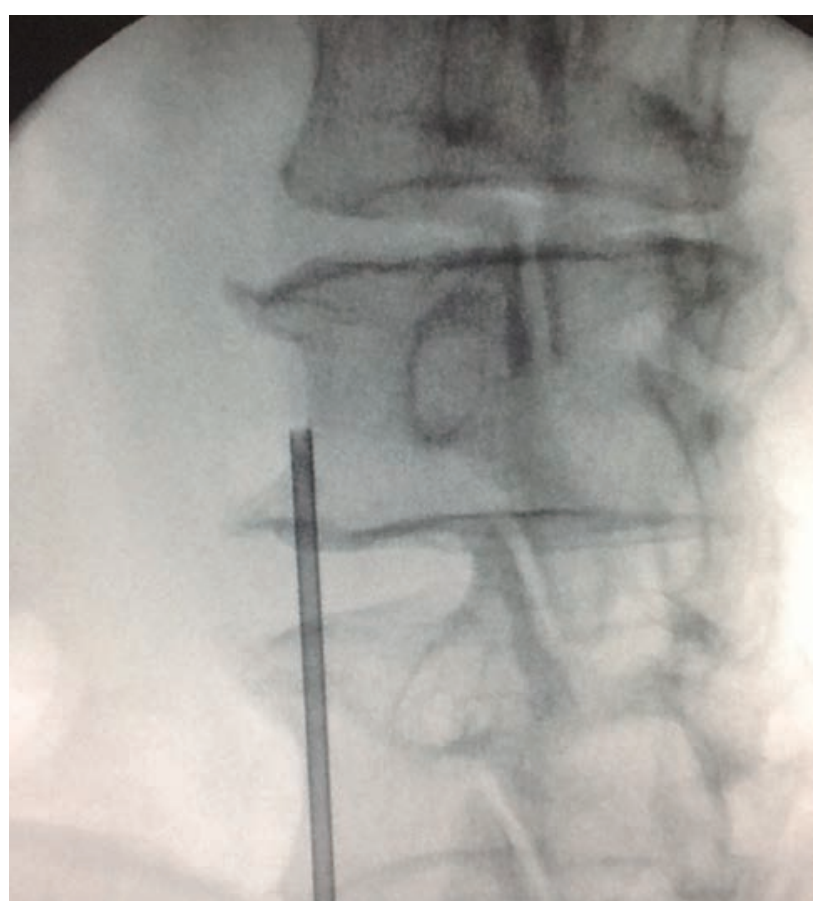

Fig. 4. Punto de entrada en visión oblicua Rx.

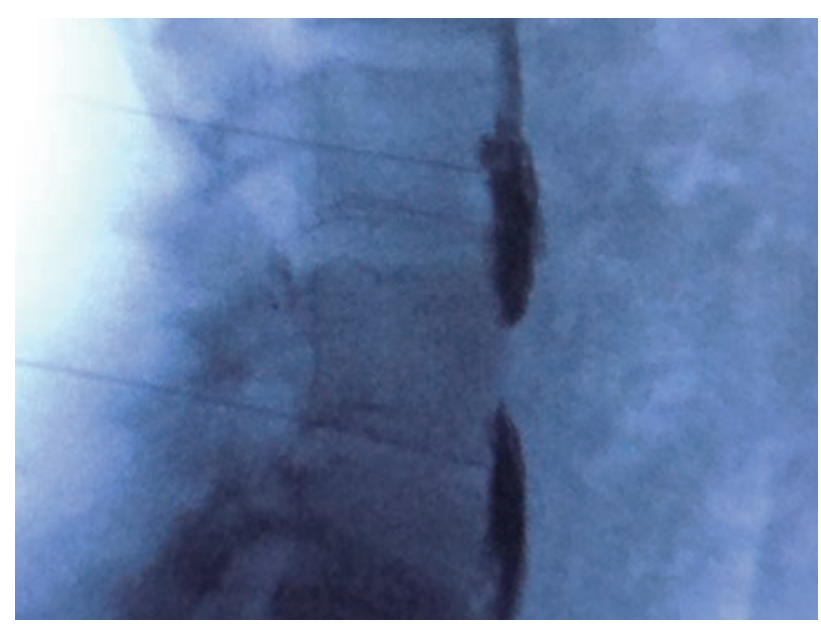

Fig. 5. Administración de contraste en visión lateral Rx.

\section{TIPOS DE BLOQUEOS}

El BSL constituye una pieza clave en el tratamiento del dolor crónico oncológico y no oncológico, especialmente en síndromes de dolor pélvico y dolor isquémico de miembros inferiores. Clásicamente se distinguen bloqueos de la cadena simpática lumbar diagnóstico-terapéuticos. Con frecuencia separar estas vertientes es complejo pues el efecto terapéutico y diagnóstico se superponen. Por este motivo se pueden dividir en bloqueos simpáticos neurolíticos y no neurolíticos (23-26).

\section{Bloqueos no neurolíticos}

$\mathrm{Su}$ objetivo primario es determinar la implicación del sistema nervioso simpático en el proceso a tratar. Con la técnica de lateral de Reid solo sería necesario colocar una aguja a nivel del ganglio simpático de L2 o L3 ya que la cadena simpática lumbar se encuentra en un espacio virtual retroperitoneal. De este modo un producto inyectado en la parte anterolateral de un cuerpo vertebral difunde por todo el espacio virtual. El volumen habitual debe ser de 10 o $15 \mathrm{ml}$ de lidocaína al $1 \%$. Aunque con frecuencia se emplean anestésicos locales de corta duración Waldman sugiere que el empleo de bupivacaína al 0,375 \% dota al paciente de un tiempo suficiente para valorar la eficacia del bloqueo simpático y su efecto sobre el dolor (27). El volumen del agente anestésico debe ser de 10 o $15 \mathrm{ml}$.

\section{Bloqueos neurolíticos}

Cuando el objetivo es mantener el efecto de la simpaticolisis de forma prolongada tras un bloqueo pronóstico positivo se realizarán bloqueos neurolíticos. Distinguimos las neurólisis químicas, mediante alcohol o fenol, y las térmicas, por radiofrecuencia. 


\section{Neurólisis química}

El fenol es un agente neurolítico con propiedades de anestésico local, hiperbárico con respecto al líquido cefalorraquídeo. A temperatura ambiente concentraciones superiores al 6,7\% requieren la adición de glicerina para asegurar su solubilidad. Las soluciones de fenol en glicerina son viscosas e hiperecogénicas aunque impiden la administración a través de catéter epidural permiten controlar su difusión mediante ecografía $(27,28)$.

El alcohol etílico es otro agente neurolítico comúnmente empleado. Es hipobárico con respecto al líquido cefalorraquídeo. Su mecanismo de acción neurolítica se debe a la potente deshidratación del tejido nervioso con la extracción de lípidos de la membrana y desnaturalización de proteínas.

En la neurólisis química se utilizarán al menos dos agujas a nivel de los ganglios simpáticos elegidos para evitar la presión excesiva en el punto de inyección ya que esto ocasionaría la extensión no deseada del agente neurolítico. Con el mismo fin el paciente permanecerá en decúbito lateral durante 10 minutos, para evitar la difusión lateral hacia el nervio genitocrural o posterior a través entre la hendidura del origen del psoas y del túnel fibroso, ocupado por los ramos comunicantes, hacia las raíces nerviosas somáticas. Eliminaremos la presencia de agentes neurolíticos de las agujas mediante la inyección de $1 \mathrm{ml}$ de aire e inyección continua de suero fisiológico durante la extracción de cada aguja.

\section{Neurólisis térmica mediante radiofrecuencia}

Las lesiones por radiofrecuencia (RF) se utilizan desde que en 1931 Kirschner introduce la diatermocoagulación del ganglio de Gasser en el tratamiento de la neuralgia de trigémino. Este método es perfeccionado después por diferentes autores (Sweet and Mark, 1953; Husperger and Wiss, 1953). El primer generador de RF fue comercializado a finales de los años 50 por S. Aranow y B.J. Cosman (29).

La inducción de lesiones por radiofrecuencia supone el paso de una corriente de frecuencia muy elevada través de una sonda termopar de $27 \mathrm{G}$; esta sonda se introduce en una cánula especial de $22 \mathrm{G}$ que está totalmente aislada salvo en la punta llamado polo activo. La radiofrecuencia convencional trata de interrumpir la conducción sensorial. La punta del electrodo debe ser colocada lo más cerca posible del punto diana. Se aplica una lesión por calor de entre $60-82{ }^{\circ} \mathrm{C}$. Pretendemos interrumpir la conducción nerviosa mediante calor o impulsos electromagnéticos de una manera continuada, como una neurólisis.

Dependiendo de las características y localización del dolor nuestros objetivos serán los ganglios simpáticos de L2, si el dolor se localiza fundamentalmente en la zona lumbar, L3, si además el dolor se extiende a la zona superior de los miembros inferiores, y L4, si el dolor es pélvico o lumbar. Habitualmente se realizan dos lesiones en L2, L3 si la patología es proximal y L3, L4 en patología distal. La localización de los ganglios simpáticos lumbares es predecible en L2 (tercio inferior del cuerpo vertebral) y L3 (tercio superior) (23-25).

Antes de realizar las lesiones por radiofrecuencia se procederá a realizar estimulación motora $(2 \mathrm{~Hz}$ y $3 \mathrm{~V})$ sensitiva $(50 \mathrm{~Hz}$ y $0,5-1 \mathrm{~V})$. Comprobaremos la ausencia de fasciculaciones o parestesias en miembros inferiores $(25,29)$.

\section{Ventajas de neurólisis térmica por radiofrecuencia}

- La lesión es controlable.

- Se puede repetir y no existe riesgo de extensión incontrolada del agente neurolítico.

- El riesgo de hipotensión es menor que en otros procedimientos simpaticolíticos.

- La incidencia de neuralgia genitofemoral es menor.

\section{SIGNOS DE EFICACIA DEL BLOQUEO}

Aunque existan signos objetivos de bloqueo simpático, otros signos como la presencia de alodinia, disestesias, la hiperalgesia o anestesia dolorosa pueden requerir una exploración exhaustiva en las que herramientas de valoración del dolor neuropático (test de Lanss, DN4 o MNSI) tienen un importante papel. El efecto placebo en pacientes agradecidos y las grandes dosis de anestésico local que provocan una inhibición central de la nocicepción pueden dificultar la interpretación del bloqueo simpático diagnóstico $(30,31)$.

Entre los signos objetivos de bloqueo simpático destacamos:

- Dilatación venosa.

- Dilatación arterial con disminución del tiempo de llenado capilar debido a la caída de las resistencias regionales sin la modificación de la presión de perfusión.

- Disminución del retorno venoso con descenso del gasto cardiaco y presión arterial si el bloqueo es extenso y bilateral.

- Aumento de la temperatura cutánea y sensación de calor en la extremidad.

- Desaparición del dolor en reposo.

\section{COMPLICACIONES}

- Como en cualquier bloqueo pueden aparecer hematomas o infección secundarios a la punción o síncopes vasovagales.

- Lumbalgia: secundaria a punción de musculatura paravertebral. Suele desaparecer en pocos días y ceder con medidas físicas y analgésicos habituales. 
- Inyección intravascular que puede deparar consecuencias como la isquemia espinal secundaria a la inyección accidental de la arteria de Adamkiewicz o una de sus ramas colaterales. Se recomienda la inyección de contraste bajo escopia continua y utilizar dosis test cuando se empleen anestésicos locales.

- Inyección subaracnoidea y cefalea pospunción dural. No siempre el LCR fluye con facilidad a través de la aguja, en ocasiones se puede puncionar un receso dural lateral. Es necesario asegurar con escopia la posición final de las agujas.

- Perforación del disco intervertebral. La sensación al perforar el disco describe como la de "atravesar un queso suizo". Si existe extrusión del material discal será lateral y lejana al canal medular por lo que no existirá peligro de compromiso neurológico. Existe la posibilidad de desencadenar discitis química o infecciosa.

- Traumatismo renal y ureteral, si el punto de punción es más de $8 \mathrm{~cm}$ distal a la línea media. Habitualmente las consecuencias son de poca magnitud aunque existen casos descritos de necrosis ureteral tras la administración de alcohol en el curso de un bloqueo neurolítico.

- Bloqueo de un nervio somático. Puede ocurrir si la aguja se coloca muy lateral y posterior al músculo psoas. El paciente referirá entumecimiento unilateral en región inguinal o área del cuádriceps. Puede aparecer hiperestesia en el área del dermatoma L1. La lesión de una raíz se presentará como un dolor intenso de características neuropáticas que puede durar hasta 5 semanas debiendo ser tratado según protocolo habitual.

- Lesión del nervio genitofemoral que es un nervio mixto, motor y sensitivo; inerva la región genital y la porción interna y superior del muslo. Surge del plexo lumbar a partir de las raíces nerviosas L1 y L2. La lesión es más frecuente en los niveles L4 y L5 donde el nervio perfora la fascia del psoas y discurre muy próximo a la cadena simpática. La incidencia es aproximadamente del $5 \%$ cuando el bloqueo se realiza con una sola aguja.

- La lesión permanente del nervio femorocutáneo ha sido descrita en un bloqueo selectivo del ganglio L3 guiado mediante TAC debido a difusión del agente neurolítico (32).

- Eyaculación retrógrada o posible e impotencia. Aparece si se realiza un bloqueo simpático bilateral.

\section{CORRESPONDENCIA:}

José Manuel González Mesa

Unidad Atención Integral del Dolor

Hospital Virgen de la Victoria

Campus de Teatinos, $\mathrm{s} / \mathrm{n}$

29010 Málaga

e-mail: josemanuelgonzalezmesa@gmail.com

\section{BIBLIOGRAFÍA}

1. Martínez-Pérez MJ, Franco-Grande A, Unzueta-Merino MC, Hervás-Puyal C, Villar-Landeira J. La simpatectomía española de René Leriche. Angiol 2005;57(6):451-6.

2. Huang J. Letter to the Editor: The history of chemical lumbar sympathectomy. The Internet Journal of Anesthesiology 2003;7(1). DOI: 10.5580/1ac1

3. Boas R. Sympathetic nerve blocks: In search of a role. Reg Anesthesia Pain Med 1998;223:292-305.

4. Schmid MR, Kissling RO, Curt A, Jaschko G, Hodler J. Sympathetic skin response: Monitoring of CT-guided lumbar sympathetic blocks. Radiology 2006;241(2):595-602.

5. Bhatnagar S, Khanna S, Roshni S, Goyal GN, Mishra S, Rana SP, et al. Early ultrasound-guided neurolysis for pain management in gastrointestinal and pelvic malignancies: An observational study in a tertiary care center of urban India. Pain Pract 201212(1):23-32.

6. Kirvelä O, Svedström E, Lundbom N. Ultrasonic guidance of lumbar sympathetic and celiac plexus block: A new technique. Reg Anesth 1992;17(1):43-6.

7. Rocco AG, Palombi D, Raeke D. Anatomy of the lumbar sympathetic chain. Reg Anesth 1995;20(1):13-9.

8. Hanna MH, Peat SJ, D'Costa F. Lumbar plexus block: An anatomical study. Anaesthesia 1993;48(8):675-8.

9. Feigl GC, Kastner M, Ulz H, Breschan C, Pixner T, Dreu $\mathrm{M}$, et al. The lumbar sympathetic trunk: Its visibility and distance to two anatomical and marks. Surg Radiol Anat 2013;35(2):99-106. DOI: 10.1007/s00276-012-1015

10. Guererin P, Obeid I, Bourghli A, Masquefa T, Luc S, Gille $\mathrm{O}$, et al. The lumbosacral plexus: Anatomic considerations for minimally invasive retroperitoneal transpsoas approach. Surg Radiol Anat 2012;34(2):151-7.

11. Ferrandiz M, Catalá E. Bloqueos simpáticos. En: Aliaga L, Catalá E, García Muret A, Masdeu J, Tornero C, editores. Anestesia Regional. $3^{\mathrm{a}}$ ed. Barcelona: Publicaciones Permanenyer; 2006 .p. 573-96.

12. Gulati A, Khelemsky Y, Loh J, Puttanniah V, Malhotra V, Cubert K. The use of lumbar sympathetic blockade at L4 for management of malignancy-related bladder spasms. Pain Physician 2011;14(3):305-9.

13. Chaturvedi A, Dash HH. Sympathetic blockade for the relief of chronic pain. J Indian Med Assoc 2001;99(12):698-703.

14. Van Eijs, Stanton-Hicks M, Van Zundert J, Faber CG, Lubenow TR, Mekhail N, et al. Evidence-based interventional pain medicine according to clinical diagnoses. Complex regional pain syndrome. Pain Practice 2011;11(1):70-87.

15. Yoshida WB, Cataneo DC, Bomfim GA, Hasimoto E, Cataneo AJ. Chemical lumbar sympathectomy in plantar hyperhidrosis. Clin Auton Res 2010;20(2):113-5.

16. Kim WO, Yoon KB, Kil HK, Yoon DM. Chemical lumbar sympathetic block in the treatment of plantar hyperhidrosis: A study of 69 patients. Dermatol Surg 2008;34(10):1340-5.

17. Rigaud J, Delavierre D, Sibert 1, labat JJ. Sympathetic nerve block in the management of chronic pelvic and perianal pain. Prog Urol 2010;20(12):1124-31 .

18. Woo JH, Park HS, Kim SC, Kim YH. The effect of lumbar sympathetic ganglion-block on gynecologic cancer-related lymphedema. Pain Physician 2013;16(4):345-52.

19. Straube S, Derry S, Moore R, McQuay H. Simpatectomía cervicotorácica o lumbar para el dolor neuropático y el síndrome de dolor regional complejo. Cochrane Database of Systematic Reviews 2010 Issue 7. Art. No.: CD002918. 
20. Van Eijs, Stanton-Hicks M, Van Zundert J, et al. Complex regional pain syndrome. En: Van Zundert J , Patijn J, Craig T, Hartrick C, lataster A, Huygen F, et al., editors. Evidence-based medicine accordind to clinical diagnoses. Oxford: Wiley-Blackwell; 2012. p. 123-5.

21. Kirvelä O, Svedström E, Lundbom N. Ultrasonic guidance of lumbar sympathetic and celiac plexus block: A new technique. Reg Anesth 1992;17(1):43-6.

22. Tang YZ, Ni JX, An JX. Complex regional pain syndrome type I following disc TRODE radiofrequency treated with continuous lumbar sympathetic trunk block using patient-controlled analgesia. Pain Med 2013;14(2):30910.

23. Ferrandiz M, Catalá E. Bloqueos simpáticos. En: Aliaga L, Catalá E, García Muret, Masdeu J, Tornero C, editores. Anestesia Regional. $3^{\mathrm{a}}$ ed. Barcelona: Publicaciones Permanenyer; 2006. p. 573-96.

24. Interventional pain procedures in the lumbar region. En: Prithvi R, Serdar E, editors. Pain relieving procedures. Oxford: Wiley-Blackwell; 2012. p.275-336.

25. Reig E, Abejón, Contreras R, Insausti J, Del Pozo C D. Simpatectomía L2-L3-L4. En: Reig E, Abejón D, Contreras R, Insausti J, Del Pozo C, editores. Manual de técnicas de radiofrecuencia para el tratamiento del dolor. Madrid: Cuarto Escalón; 2004. p.65-72.
26. Krumova EK, Gussone C, Regeniter S, Westermann A, Zenz M, Maier C. Are sympathetic blocks useful for diagnostic purposes? Reg Anesth Pain Med 2011;36(6):560-7.

27. Stanton-Hicks M. En: Lumbar Sympathetic Nerve Block and neurolysis. En: Stanton-Hicks M.Waldman. Pain management. Vol 2. Philadelphia: Saunders Elsevier; 2007. p.1314-21.

28. Ferrandiz M, Catalá E. Bloqueo simpático lumbar. En: Aliaga L, de León Casasola O, Nebreda C, Vallejo R, editores. Técnicas intervencionistas para el tratamiento del dolor crónico. Barcelona: Glosa S.L.; 2011. p.161-9.

29. Gauci C Radiofrecuencia sobre el simpático lumbar. En: Gauci C. Manual de técnicas de radiofrecuencia. Amsterdan: Flivopress; 2004. p.101-9.

30. Manjunath PS, Jayalakshmi TS,Dureja GP, Prevost AT. Management of lower limb complex regional pain syndrome type 1: An evaluation of percutaneous radiofrequency thermal lumbar sympathectomy versus phenol lumbar sympathetic neurolysis -a pilot study . Anesth Analg 2008;106(2):647-9.

31. Park SY, Nahm FS, Kim YC, Lee SC, Sim SE, Lee SJ. The cut-off rate of skin temperature change to confirm successful lumbar sympathetic block. J Int Med Res2010;38(1):266-75.

32. Pennekamp W, Krumova EK, Feigl GP, Frombach E, Nicolas $\mathrm{V}$, Schwarzer A, et al. Permanent lesion of the lateral femoral cutaneous nerve after low-volume ethanol $96 \%$ application on the lumbar sympathetic chain. Pain Physician 2013;16(4):391-7. 